LINKS:

Plasmodium berghei

http://www.ncbi.nlm.nih.gov/entrez/query.

fcgi?db=genomeprj\&cmd=Retrieve\&dopt=Overvi

ewElist_uids $=9536$
MALARIA

\section{Plasmodium develops in lymph nodes}

When a mosquito infected with Plasmodium, the parasite that causes malaria, bites a mammal, the parasite travels to the animal's liver. Once in the liver, the parasites can fully develop, burst out of the liver cells and infect erythrocytes, leading to the onset of malaria.

Until now, the liver was thought to be the only place that parasites could develop. However, a recent paper published in Nature Medicine on the first quantitative real-time imaging study of the movements of Plasmodium through mammalian tissue reports that sporozoites can partially develop in lymph nodes.

Robert Ménard and his colleagues at the Pasteur Institute in Paris used epifluorecence time-lapse microscopy, Anopheles stephensi mosquitoes and clones of the rodent-infecting Plasmodium berghei that express green fluorescent protein to analyse the in vivo behaviour of sporozoites in several rodent species. They infected the mosquitoes with $P$. berghei, and then allowed them to bite a mouse. For each mosquito bite, $\sim 20$ parasites were injected into the mouse's skin. Injected parasites were found to move forward through the skin in an apparently random path.

Most parasites leaving the skin went on to invade blood vessels, and so make their way to the liver. However, about 25\% invaded lymphatic vessels and ended up in the lymph nodes closest to the bite. Within four hours of the mosquito bite, most parasites in the lymph nodes were being degraded by dendritic leukocytes, but some escaped degradation and began to develop into exoerythrocytic forms, which was thought to only happen in liver cells. Fifty-two hours after the mosquito bite, however, no parasites remained in the lymph nodes, which suggests that they cannot develop completely there.

Although this previously unrecognized step of the Plasmodium life cycle probably does not contribute to the appearance of malaria symptoms, it could influence the immune response of the host, and might therefore have implications for vaccine strategies.

Annie Tremp

\section{REFERENCES AND LINKS}

ORIGINAL RESEARCH PAPER Amino, R. et al. Quantitative imaging of Plasmodium transmission from mosquito to mammal. Nature Med. 22 Jan 2006 (doi: 10.1038/nm1350) MOVIES Several movies by Amino et al. showing sporozoite movement in the skin can be found online at: http://www.nature.com/nm/journal/vaop/ncurrent/suppinfo/ nm1350_S1.html
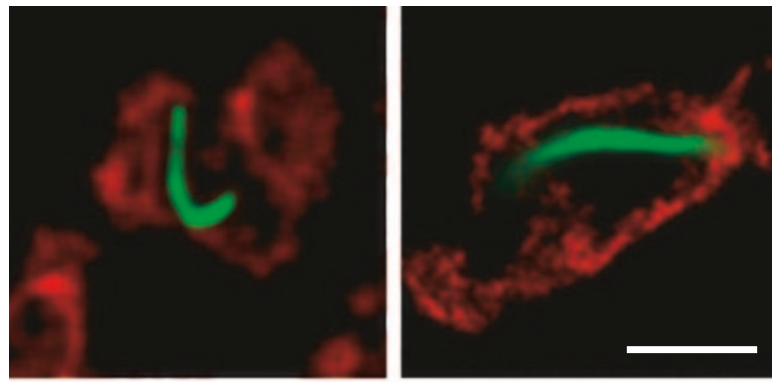

Lymph-node sporozoites (green) interact with dendritic cells labelled with antibodies to CD11c (red) $4 \mathrm{~h}$ after injection. Images reproduced with permission from Amino et al. @ (2006) Macmillan Publishers Ltd. Scale bar represents $10 \mu \mathrm{m}$. 